\title{
Field-dependent photoelectron extraction from CsI in different gases
}

\author{
A.Breskin, A.Buzulutskov and R.Chechik \\ Department of Particle Physics \\ The Weizmann Institute of Science, 76100 Rehovot, Israel \\ A.DiMauro and E.Nappi \\ INFN, Sez. Bari and Politecnico, 70100 Bari, Italy \\ G.Paic * \\ SUBATECH, Nantes, France \\ F.Piuz \\ CERN, Geneva, Switzerland
}

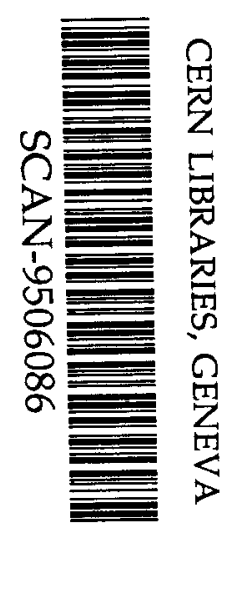

\begin{abstract}
We present the results of our latest investigations, made in laboratory and in a $\mathrm{RICH}$ detector, of the dependence of the UV quantum efficiency (QE) of CsI on the gas and the electric field. We confirm that in a charge collection mode the $Q E$ in a gas is generally lower than that in vacuum, due to electron backscattering; in a charge multiplication mode the $\mathrm{QE}$ increases with the field and reaches the vacuum value at high gas gains. The lowest photoemission under low electric field at the photocathode surface was observed in He-based gas mixtures; in Ar-hydrocarbon mixtures it approaches that of pure hydrocarbons. In $\mathrm{CH}_{4}$ and in $\mathrm{CH}_{4} / \mathrm{i}-\mathrm{C}_{4} \mathrm{H}_{10}$ the $\mathrm{QE}$ value is practically independent of the field and is close to that in vacuum. The implications to CsI-based UV-detectors, particularly to fast RICH devices, are discussed.
\end{abstract}

Presented at the Wire Chamber Conference, Vienna, Austria, Feb 13-17, 1995

To be published in Nuclear Instruments and Methods in Physics Research A

*also Rugier Boskovic Institute, Zagreb, Chroatia 


\section{Introduction}

Recent investigations suggest that UV-photon imaging detectors based on CsI solid photoconverters coupled to gaseous electron multipliers, could be adequate for the Ring Imaging Cherenkov (RICH) technique [1, 2, 3, 4]. The quantum efficiency (QE) of the CsI photocathode is one of the major parameters in this technique. It was recently demonstrated that the electron extraction probability from CsI is strongly dependent on the gas and on the electric field $[5,6]$.

Already 40 years ago it was recognized that once escaped from the photocathode the photoelectrons diffuse back, even in the presence of an electric field, due to elastic collisions with the gas molecules [7]. In particular, it was observed that the backscattering contribution is large for monoatomic, noble gases and can be very small for some molecular gases such as $\mathrm{CH}_{4}$ or $\mathrm{CO}_{2}[8,9,10,11,12]$.

The results mentioned in the literature were obtained in a charge collection mode, and only recently photoelectron extraction efficiency under gas multiplication has been investigated in some hydrocarbon gases: $\mathrm{CH}_{4}, \mathrm{C}_{2} \mathrm{H}_{6}$ and $\mathrm{i}-\mathrm{C}_{4} \mathrm{H}_{10}$, in the pressure range 20-60 Torr [5]. It was demonstrated that the QE of CsI as a function of the electric field is rather constant in the charge collection mode, and has a value depending on the nature of the gas. In the gas multiplication mode it enhances in coherence with the gas multiplication commencement. It was clearly observed that the $\mathrm{QE}$ in all gases studied approaches the $\mathrm{QE}$ value in vacuum, under high gas multiplication. In $\mathrm{CH}_{4}$, the $\mathrm{QE}$ is rather constant and reaches $97 \%$ of the vacuum value at low fields, in the collection mode. This behaviour is explained by a reduction of the elastic backscattering due to dominating inelastic processes, i.e. ionization and excitation, in the multiplication region. Indeed, the electron direction of motion is only slightly perturbed by inelastic collisions, in contrary to the randomization of the velosity vector in elastic collisions. It was predicted that any gas, including noble gas mixtures, should obey such a universal behaviour [5].

In the present work, the combined effects of electric field, gas and pressure on photoelectron extraction from $\mathrm{CsI}$ are further investigated at higher pressures and in a variety of gas mixtures: $\mathrm{CH}_{4}, \mathrm{CH}_{4} / \mathrm{i}-\mathrm{C}_{4} \mathrm{H}_{10}, \mathrm{He} / \mathrm{CH}_{4}, \mathrm{He} / \mathrm{i}-\mathrm{C}_{4} \mathrm{H}_{10}, \mathrm{Ar} / \mathrm{CH}_{4}$ and $\mathrm{Ar} / \mathrm{i}-\mathrm{C}_{4} \mathrm{H}_{10}$, in the pressure range 200-800 Torr. Present laboratory measurements were compared to photoelectron counting measurements in a RICH detector.

\section{Experimental setup and procedure}

The systematic measurements of the dependence of the CsI quantum efficiency on the electric field and on the gas and pressure were carried out in the laboratory, both with a single photon counting and in a DC current technique. The results in some mixtures were compared with those measured in a CsI-based wire chamber, in a RICH experiment.

The experimental setup was similar to that described elsewhere [5], replacing the twostage gas amplification device by a three-stage one. The latter provided more stable operation at higher pressures. The experimental device consists of a two-stage parallelplate chamber coupled to a multiwire proportional element. The CsI photocathode acts as 
a source of photoelectroncs and a cathode of the first parallel-plate amplification element. The flux of UV-photons from a pulsed $\mathrm{H}_{2}$ lamp in air was reduced to a single photon level. The average wavelength of photons hitting the photocathode is estimated to be about 190 $\mathrm{nm}$. The measured value was the number of photoelectrons per UV-light pulse, which is proportional to the $\mathrm{QE}$ value. The procedure of the determination of this number is described in [5].

The measurements in the DC current mode were made with a photocathode coupled to a single mesh electrode. The pulsed lamp was replaced by an intense continuous flux lamp, either that of $\mathrm{Hg}(\mathrm{Ar})$ or $\mathrm{D}_{2}$, with a selected wavelength of $185 \mathrm{~nm}$. A picoamperemeter was connected to the photocathode. For each gas mixture the UV-induced photocurrent was measured first in gas and then in vacuum at the same absolute electric field values. The DC current technique was used to estimate the gas gain as well.

Other details of the apparatus and of the measurement procedure are presented in ref. $[5]$.

The QE of large area photocathodes $\left(30 \times 30 \mathrm{~cm}^{2}\right)$ was evaluated using a RICH detector in a proximity focusing geometry as discussed in detail in ref. [3, 4]. The photosensitive CsI layer was deposited on the cathode of a wire chamber, segmented into pads, providing a 2-dimensional localization of the photoelectrons. The $\mathrm{QE}$ of the photocathode is obtained using the Frank-Tamm formula, on the basis of the number of counted photoelectrons per Cherenkov ring, taking into account the experimental conditions [4].

\section{Results}

\subsection{Laboratory measurements}

Fig. 1 shows the relative $\mathrm{QE}$ and the gas gain for $\mathrm{He} / \mathrm{CH}_{4}(95 / 5)$ and $\mathrm{He} / \mathrm{i}-\mathrm{C}_{4} \mathrm{H}_{10}(95 / 5)$ at 800 Torr, as a function of the reduced electric field, $\mathrm{E} / \mathrm{p}$, at the photocathode surface. At low fields in the charge collection mode, the $\mathrm{QE}$ increases and reaches a plateau at $\mathrm{E} / \mathrm{p}=2$ $\mathrm{V} / \mathrm{cm}$.Torr, in accordance with the data from [11] for the same gas mixture. When the gas multiplication starts, approximately at $\mathrm{E} / \mathrm{p}=5 \mathrm{~V} / \mathrm{cm} \cdot$ Torr, the $\mathrm{QE}$ increases again and reaches a second plateau, at a gain value of the order of 100 . The QE data points are normalized to the value at this second plateau, obtained by averaging over the three last points. As one can see from the figure, the ratio between the $\mathrm{QE}$ values at the two plateaux is $50 \%$ in $\mathrm{He} / \mathrm{CH}_{4}$ and $40 \%$ in $\mathrm{He} / \mathrm{i}-\mathrm{C}_{4} \mathrm{H}_{10}$.

Fig. 2 shows, for the same gas mixtures and pressure, the ratio of the photocurrent in gas to that in vacuum. The value of this ratio in the middle of the charge collection plateau, around $\mathrm{E} / \mathrm{p}=2.5 \mathrm{~V} / \mathrm{cm}$. Torr, ammounts to about $45 \%$ for both mixtures. This is very close to the ratio of the $\mathrm{QE}$ in the charge collection mode to that in the multiplication, obtained with the photon counting technique (see above, Fig.1). This fact, established now for a noble gas-based mixture, supports our statement [5], that the QE value in gas in multiplication mode approaches the $\mathrm{QE}$ value in vacuum.

The Ar-based gas mixtures were studied with the DC current technique only. Fig.3 shows the ratio of the photocurrent in gas to that in vacuum, measured in $\mathrm{Ar}, \mathrm{Ar} / \mathrm{CH}_{4}$ 
$(80 / 20), \mathrm{Ar} / \mathrm{CH}_{4}(50 / 50)$ and $\mathrm{Ar} / \mathrm{i}-\mathrm{C}_{4} \mathrm{H}_{10}(80 / 20)$ at 770 Torr. The photocurrent in pure Ar at the end of the charge collection plateau is about $40 \%$ of that in vacuum. It is in accordance with the literature $[8,10,12]$ and by a factor of $2-3$ larger compared to that observed in pure $\mathrm{He}[9,12]$. The lower backscattering in Ar-based mixtures results in photocurrents in the charge collection mode reaching $70-80 \%$ and $90 \%$ of the vacuum value in $\mathrm{Ar} / \mathrm{i}-\mathrm{C}_{4} \mathrm{H}_{10}(80 / 20)$ and $\mathrm{Ar} / \mathrm{CH}_{4}(50 / 50)$, respectively. This is very close to the respective $\mathrm{QE}$ values in $\mathrm{i}-\mathrm{C}_{4} \mathrm{H}_{10}[5]$ and $\mathrm{CH}_{4}$ (see below).

It is interesting that in $\mathrm{Ar} / \mathrm{i}-\mathrm{C}_{4} \mathrm{H}_{10}$ the gas multiplication starts earlier than in $\mathrm{Ar} / \mathrm{CH}_{4}$, in contrast to He-based mixtures (see Fig.2). This observation can be explained by the Penning effect [13]: the ionization potential of $\mathrm{i}-\mathrm{C}_{4} \mathrm{H}_{10}$ is lower than the excitation levels of Ar, leading to an additianal channel for the avalanche development.

Fig. 4 shows the relative $\mathrm{QE}$ and the gas gain in $\mathrm{CH}_{4}$ as a function of the reduced electric field, measured at 200, 400 and 800 Torr. The measurements at 800 Torr are limited to the low $\mathrm{E} / \mathrm{p}$ values because of the chamber instability at higher fields.

Fig.5 shows the relative $\mathrm{QE}$ and the gas gain in $\mathrm{CH}_{4} / \mathrm{i}-\mathrm{C}_{4} \mathrm{H}_{10}(95 / 5)$ at 200 and 400 Torr. This mixture is favorable for application in photon imaging detectors due to its better quenching properties as compared to pure $\mathrm{CH}_{4}$. The rather flat relative QE graph indicates that this mixture preservs the advantage of $\mathrm{CH}_{4}$.

All the data points in Figs.4,5 are normalised to the QE value, averaged over the points measured at $\mathrm{E} / \mathrm{p}$ values higher than $30 \mathrm{~V} / \mathrm{cm} \cdot$ Torr (under gas gain of the order of 100). The normalization for 800 Torr $\mathrm{CH}_{4}$ is somewhat arbitrary, using the data from the photocurrent ratio measurements.

Comparing the results at 200 and 400 Torr one can deduce a very small dependence of the $\mathrm{QE}$ on the electric field, in pure methane. The dependence on pressure is small as well. The results obtained with $\mathrm{CH}_{4} / \mathrm{i}-\mathrm{C}_{4} \mathrm{H}_{10}$ mixtures indicate that the presence of isobutane, even in small percentage, decreases the $\mathrm{QE}$ in the collection plateau, but to a very small extent.

Fig. 6 shows the ratio of the photocurrent (in DC technique) in gas to that in vacuum for $\mathrm{CH}_{4}$ at 200 and 800 Torr and $\mathrm{CH}_{4} / \mathrm{i}-\mathrm{C}_{4} \mathrm{H}_{10}$ at 800 Torr. The results are in good agreement with those obtained with the photon counting technique. The dependence of the $\mathrm{QE}$ on the gas pressure is more evident compared to Figs.4,5. The photocurrent ratio in the middle of the charge collection plateau has a value of about $90 \%$ in 800 Torr $\mathrm{CH}_{4}$ and about $87 \%$ in 800 Torr $\mathrm{CH}_{4} / \mathrm{i}-\mathrm{C}_{4} \mathrm{H}_{10}$; in 200 Torr $\mathrm{CH}_{4}$ this ratio is higher, about $95 \%$, in coherence with the results obtained at 60 Torr $\mathrm{CH}_{4}[5]$.

\subsection{Measurements with RICH}

The $\mathrm{QE}$ of the photocathode was first measured in pure $\mathrm{CH}_{4}$. A potential was applied to the wire chamber, resulting in a $\mathrm{E} / \mathrm{p}$ value of around $4 \mathrm{~V} / \mathrm{cm} \cdot$ Torr at the CsI surface. Laboratory measurements (Fig.6) indicate that at such fields the QE value of the photocathode in $\mathrm{CH}_{4}$ is about $90 \%$ of the maximum nominal vacuum value. Then the detector, with the same photocathode, was circulated with different noble gas-based mixtures. The mean numbers of photoelectrons per ring were compared to that obtained with $\mathrm{CH}_{4}$. 
Table 1: The ratio $\left(\mathrm{R}_{p e}\right)$ of the number of photoelectrons measured in the gas mixture to that in $\mathrm{CH}_{4}$, at 1 atm, obtained in the $\mathrm{RICH}$ detector and the ratio $\left(\mathrm{R}_{i}\right)$ of the photocurrent at $185 \mathrm{~nm}$ measured in the gas mixture to that in $\mathrm{CH}_{4}$, obtained in the laboratory

\begin{tabular}{llll}
\hline \hline Gas mixture & $\begin{array}{l}\mathrm{E} / \mathrm{p} \\
{[\mathrm{V} / \mathrm{cm} \text {-Torr }]}\end{array}$ & $\begin{array}{l}\mathrm{R}_{p e} \\
(\mathrm{RICH})\end{array}$ & $\begin{array}{l}\mathrm{R}_{i} \\
(\mathrm{Lab})\end{array}$ \\
\hline $\mathrm{Ar} / \mathrm{i}-\mathrm{C}_{4} \mathrm{H}_{10}(80 / 20)$ & $3.0-3.1$ & $0.73-0.76$ & 0.81 \\
\hline $\mathrm{Ar} / \mathrm{CH}_{4}(80 / 20)$ & 3.1 & 0.78 & 0.93 \\
\hline $\mathrm{Ar} / \mathrm{CH}_{4}(50 / 50)$ & $3.7-3.8$ & $0.88-0.94$ & 1.0 \\
\hline $\mathrm{Ar} / \mathrm{CH}_{4} / \mathrm{i}-\mathrm{C}_{4} \mathrm{H}_{10}$ & $2.7-2.8$ & $0.82-0.86$ & - \\
$(86 / 7 / 7)$ & & & \\
\hline $\mathrm{He} / \mathrm{i}-\mathrm{C}_{4} \mathrm{H}_{10}(80 / 20)$ & 2.5 & 0.55 & - \\
\hline $\mathrm{He} / \mathrm{i}-\mathrm{C}_{4} \mathrm{H}_{10}(95 / 5)$ & 2.5 & - & 0.49 \\
\hline $\mathrm{He} / \mathrm{CH}_{4}(95 / 5)$ & 2.5 & - & 0.51 \\
\hline \hline
\end{tabular}

The detection of the Cherenkov photons was affected by particle- and photoelectron avalanche-induced secondary photon effects. In case of $\mathrm{CH}_{4}$, that background contribution was $5-7 \%$ of the Cherenkov photon yield. In $\mathrm{Ar} / \mathrm{CH}_{4}(50 / 50$ and $80 / 20)$ and $\mathrm{He} / \mathrm{i}-\mathrm{C}_{4} \mathrm{H}_{10}$ $(80 / 20)$ the background increased to a $20-30 \%$ level, making more difficult the counting of the Cherenkov photons. On the contrary, in $\mathrm{Ar} / \mathrm{i}-\mathrm{C}_{4} \mathrm{H}_{10}(80 / 20)$ that level was found similar to that in $\mathrm{CH}_{4}$. The data shown below are corrected for these secondary effects.

Compared to $\mathrm{CH}_{4}$, a decrease of the QE was observed in these mixtures, as seen in Table 1. Table 1 shows the ratio of the number of photoelectrons measured in the gas mixture to that in $\mathrm{CH}_{4}$, at $1 \mathrm{~atm}$, in the $\mathrm{RICH}$ detector. For comparison, also shown in the table is the recalculated data obtained in the laboratory: the ratio of the photocurrent at $185 \mathrm{~nm}$ in the gas mixture to that in $\mathrm{CH}_{4}$, at the reduced field values similar to that applied at the CsI surface in the wire chamber of the RICH detector. Comparing these two sets of measurements one can see a reasonable agreement between the data.

\section{Summary and conclusions}

We further investigated the electric field effects on the quantum efficiency (QE) of CsI in hadrocarbon- and noble gas-based mixtures, at pressures reaching $1 \mathrm{~atm}$. We confirm that the photoelectron extraction from solid photocathodes into gas media has a universal behaviour as a function of field. In a charge collection mode, the $\mathrm{QE}$ is lower than that in vacuum and is independent of the field. In a charge multiplication mode, the $\mathrm{QE}$ increases with the field and reaches the vacuum value at high gas gains. The most pronounced behaviour, leading to a very large difference in the QE between the collection and multiplication modes, was observed in He-based gas mixtures, while losses in QE 
observed in Ar-hydrocarbon mixtures approach that in pure hydrocarbons. In pure $\mathrm{CH}_{4}$ and in some $\mathrm{CH}_{4}$-hydrocarbon gas mixtures the $\mathrm{QE}$ is practically independent of the field and is close to that in vacuum even at low fields and at atmospheric pressure.

These results are explained by the difference in extraction efficiency of photoelectrons from CsI, which depends on the elastic backscattering probability from different gas molecules. At high gas gain elastic backscattering is taken over by inelastic collisions, resulting in a $\mathrm{QE}$ value equal to that in vacuum, independently of the gas nature. A more quantitative analysis of the results obtained will be possible after the next step of the present study, when the process of electron transport in gas mixtures will be simulated. Prelimanary results of this computer sumulation confirm the trends in the experimental results. The consequences of this study are of great relevance to the design of CsI-based wire chambers for UV-photon imaging in $\mathrm{RICH}$. They open the possibility of reducing the amount of flammable gas in very large systems, as proposed for example for the ALICE/LHC experiment. However it necessitates further studies.

This work was done within the CERN RD26 research project. We would like to thank our collegues for helpful discussions and support. We would like to thank Dr.S.Biagi for fruitfull discussions and for the interaction cross-sections in gases, used for theoretical estimation of backscatterring. This work was supported by the Basic Research Foundation of the Israel Academy of Sciences and Humanities, by the United States-Israel Binational Science Foundation (BSF), by the Israel Ministry of Science and Technology, by the Commission of the European Communities and by the Foundation Mordoh Mijan de Salonique. A.Buzulutskov is grateful to the Feinberg Graduate School for his support.

\section{References}

[1] Proc. of the First Workshop on RICH Detectors, Bari, Italy, June 2-5, 1993, Nucl. Instr. and Meth. A343(1994)1-326, and references therein.

[2] A.Braem, A.DiMauro, E.Nappi, A.Ljubicic, G.Paic, F.Piuz, F.Poza, R.S.Ribeiro, T.Scognetti, T.D.Williams, Nucl. Instr. and Meth. A343(1994)163.

[3] A.DiMauro et al., Development of a large area advanced fast RICH detector for particle identification at the LHC operated with heavy ions, Preprint CERN/DRDC 94-49, RD-26 Status Report, 1994.

[4] F.Piuz, talk at this Conference.

[5] A.Breskin, A.Buzulutskov, R.Chechik, D.Vartsky, G.Malamud and P.Miné, Nucl. Instr. and Meth. A344(1994)537.

[6] A.Buzulutskov, A.Breskin and R.Chechik, Field enhancement of the photoelectric and secondary electron emission from CsI, Preprint WIS-94/11/Feb.-PH., J. Appl. Phys. 77(5), (1995), in press. 
[7] L.B.Loeb, Basic processes of gaseous electronics, (University of California Press, Berkley, 1955), p.601.

[8] G.Charpak, W.Dominik, F.Sauli, S.Majewski, IEEE Trans. Nucl. Sci. NS-30 (1983) 134.

[9] D.F.Anderson, R.Bouclier, G.Charpak, S.Majewski and G.Kneller, Nucl. Instr. and Meth. 217 (1983) 217.

[10] J.Seguinot, G.Charpak, Y.Giomataris, V.Peskov, J.Tischhauser and T.Ypsilantis, Nucl. Instr. and Meth. A297 (1990) 133.

[11] C.Lu, K.T.McDonald and Y.Zhu, Nucl. Instr. and Meth. A334(1993)328.

[12] E.M.Gushchin, A.N.Lebedev, S.V.Somov, V.P Protasov, M.K.Timofeev and G.I.Tipografshchik, Instr. Exp. Tech. 36(1993)754.

[13] B.Sitar, G.I.Merson, V.A.Chechin and Yu.A.Budagov, Ionization measurements (Springer-Verlag, Berlin, 1993), p.81. 


\section{Figure captions}

Fig.1 The relative quantum efficiency of CsI, measured with the photon counting technique at about $190 \mathrm{~nm}$, and the gas gain, as a function of the reduced electric field in $\mathrm{He} / \mathrm{CH}_{4}(95 / 5)$ and $\mathrm{He} / \mathrm{i}-\mathrm{C}_{4} \mathrm{H}_{10}(95 / 5)$. The $\mathrm{QE}$ data points are normalized to the last three points averaged.

Fig. 2 The ratio of the photocurrent from CsI, at $185 \mathrm{~nm}$, in $\mathrm{He} / \mathrm{CH}_{4}(95 / 5)$ and $\mathrm{He} / \mathrm{i}$ $\mathrm{C}_{4} \mathrm{H}_{10}(95 / 5)$ to the photocurrent in vacuum (measured at the same absolute electric field values), as a function of the reduced electric field in gas.

Fig.3 The ratio of the photocurrent from CsI, at $185 \mathrm{~nm}$, in $\mathrm{Ar}, \mathrm{Ar} / \mathrm{CH}_{4}(80 / 20), \mathrm{Ar} / \mathrm{CH}_{4}$ $(50 / 50)$ and $\mathrm{Ar} / \mathrm{i}-\mathrm{C}_{4} \mathrm{H}_{10}(80 / 20)$ at 770 Torr to the photocurrent in vacuum (measured at the same absolute electric field values), as a function of the reduced electric field in gas.

Fig.4 The relative quantum efficiency of CsI, measured with the photon counting technique at about $190 \mathrm{~nm}$, and the gas gain, as a function of the reduced electric field in $\mathrm{CH}_{4}$ at 200, 400 and 800 Torr. For 200 and 400 Torr the QE data points are normalized to the points at $\mathrm{E} / \mathrm{p} \geq 30 \mathrm{~V} / \mathrm{cm}$.Torr, averaged.

Fig.5 The relative quantum efficiency of CsI, measured with the photon counting technique at about $190 \mathrm{~nm}$, and the gas gain, as a function of the reduced electric field in $\mathrm{CH}_{4} / \mathrm{i}-\mathrm{C}_{4} \mathrm{H}_{10}(95 / 5)$ at 200 and 400 Torr. The $\mathrm{QE}$ data points are normalized to the points at $\mathrm{E} / \mathrm{p} \geq 30 \mathrm{~V} / \mathrm{cm} \cdot$ Torr, averaged.

Fig.6 The ratio of the photocurrent from CsI, at $185 \mathrm{~nm}$, in $\mathrm{CH}_{4}$ at 200 and 800 Torr and in $\mathrm{CH}_{4} / \mathrm{i}-\mathrm{C}_{4} \mathrm{H}_{10}$ at 800 Torr to the photocurrent in vacuum (measured at the same absolute electric field values), as a function of the reduced electric field in gas. 


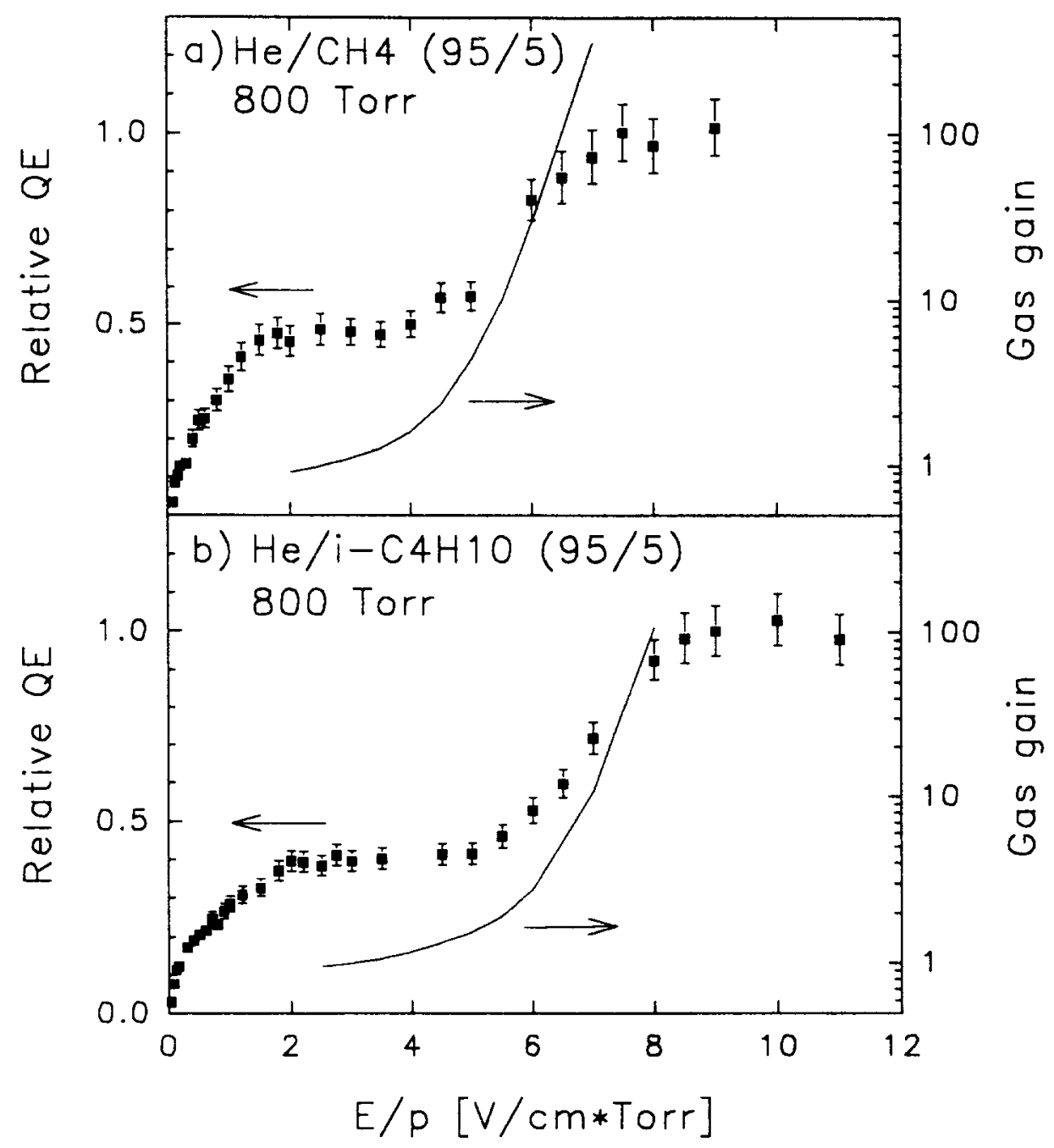

Fig.1 


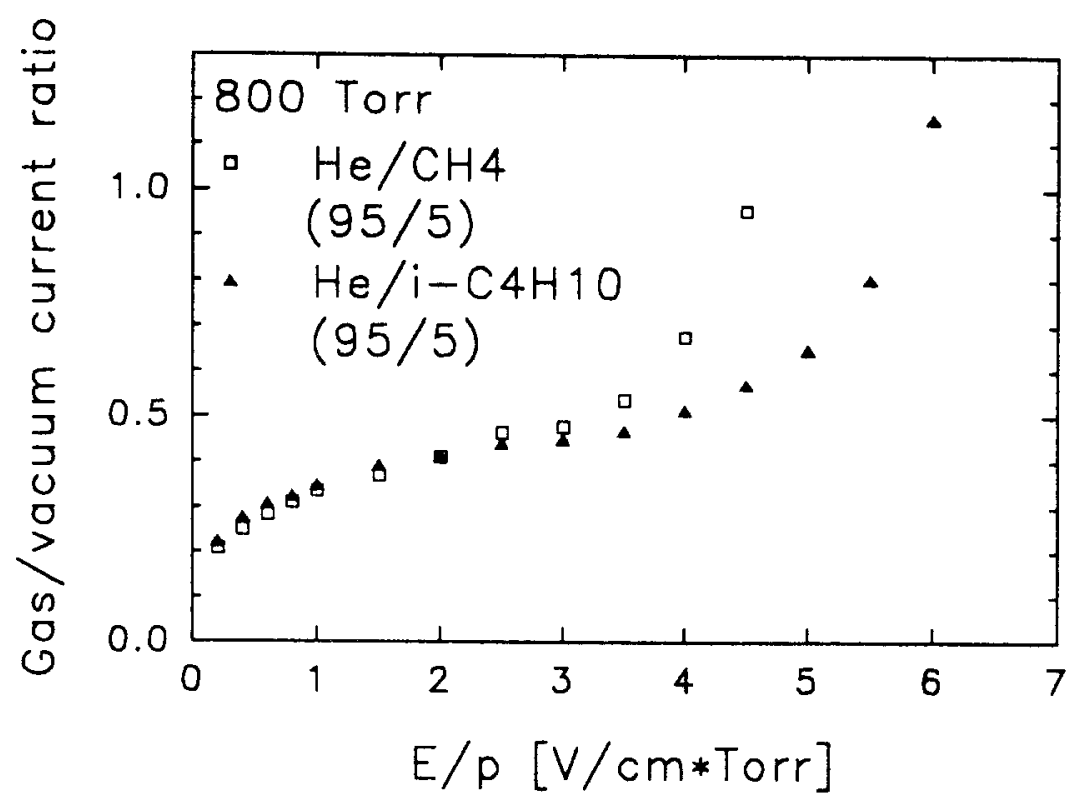

Fig.2

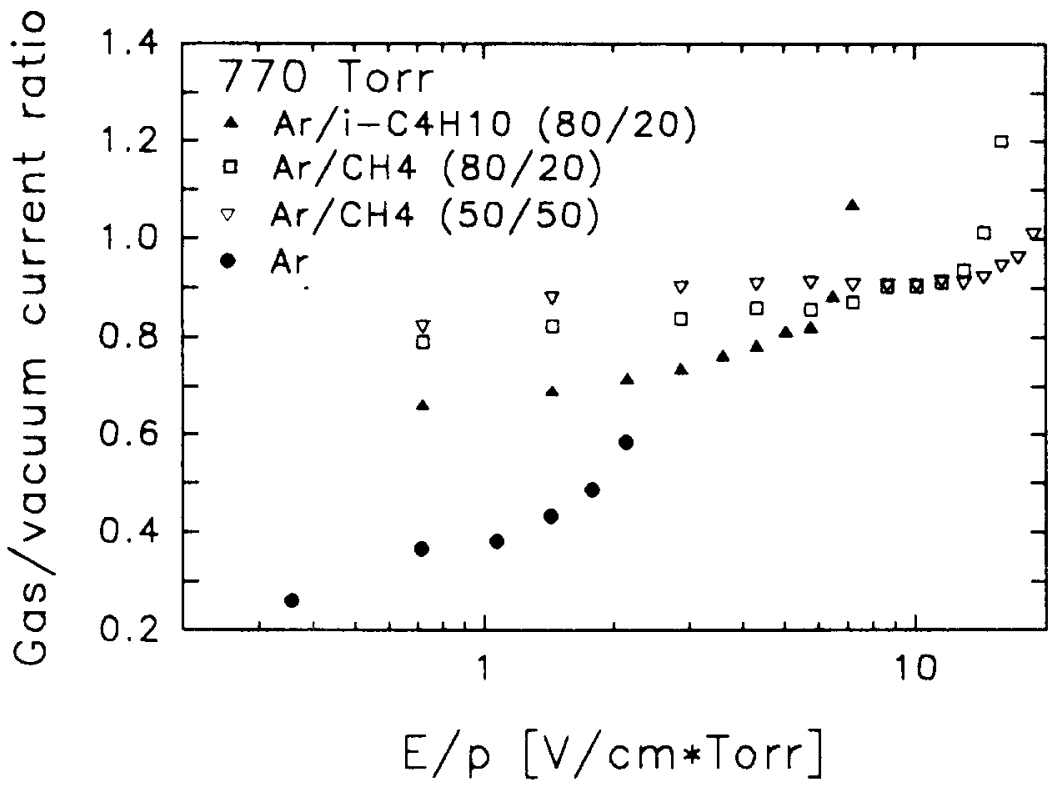

Fig.3 


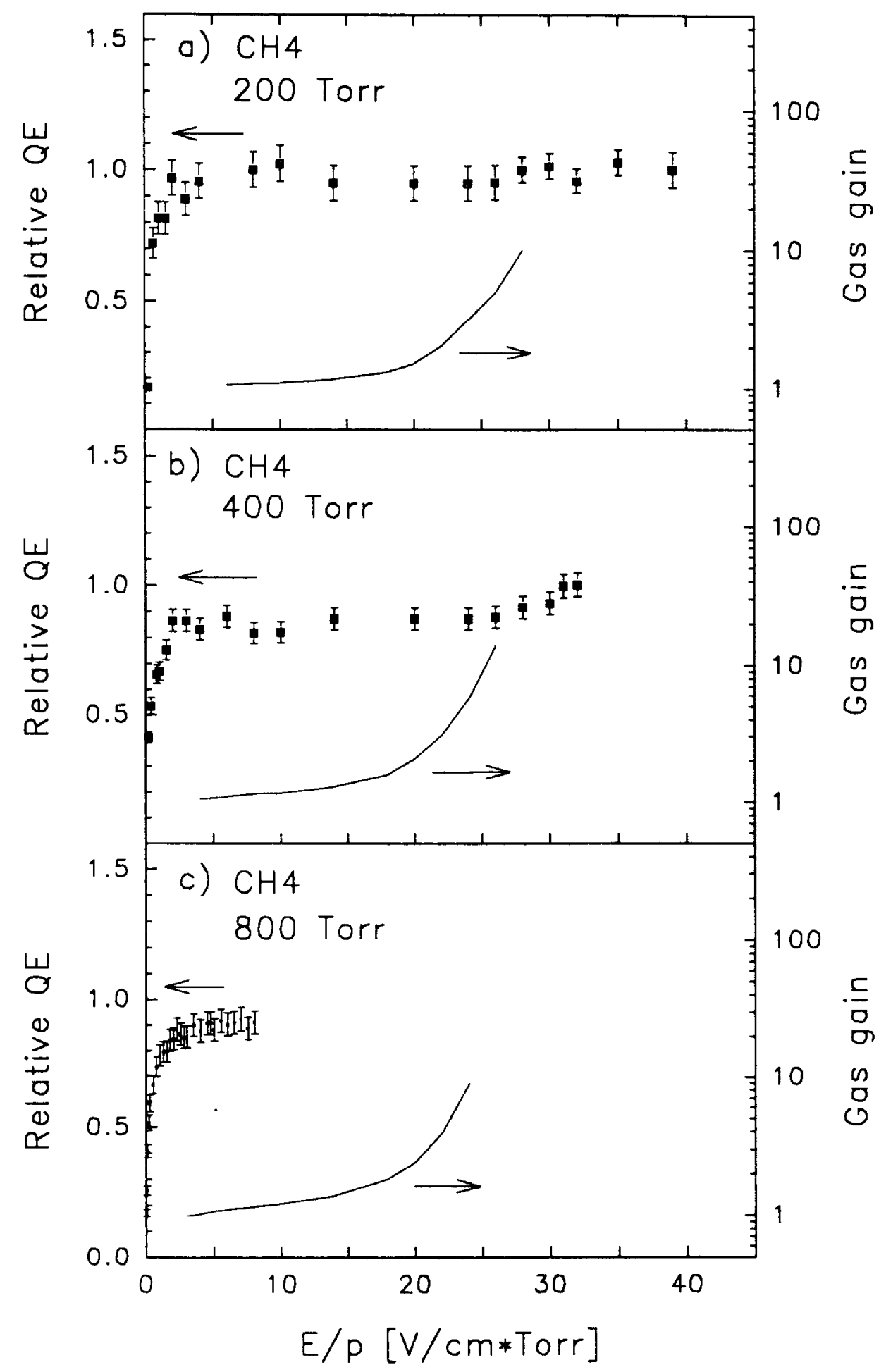

Fig.4 

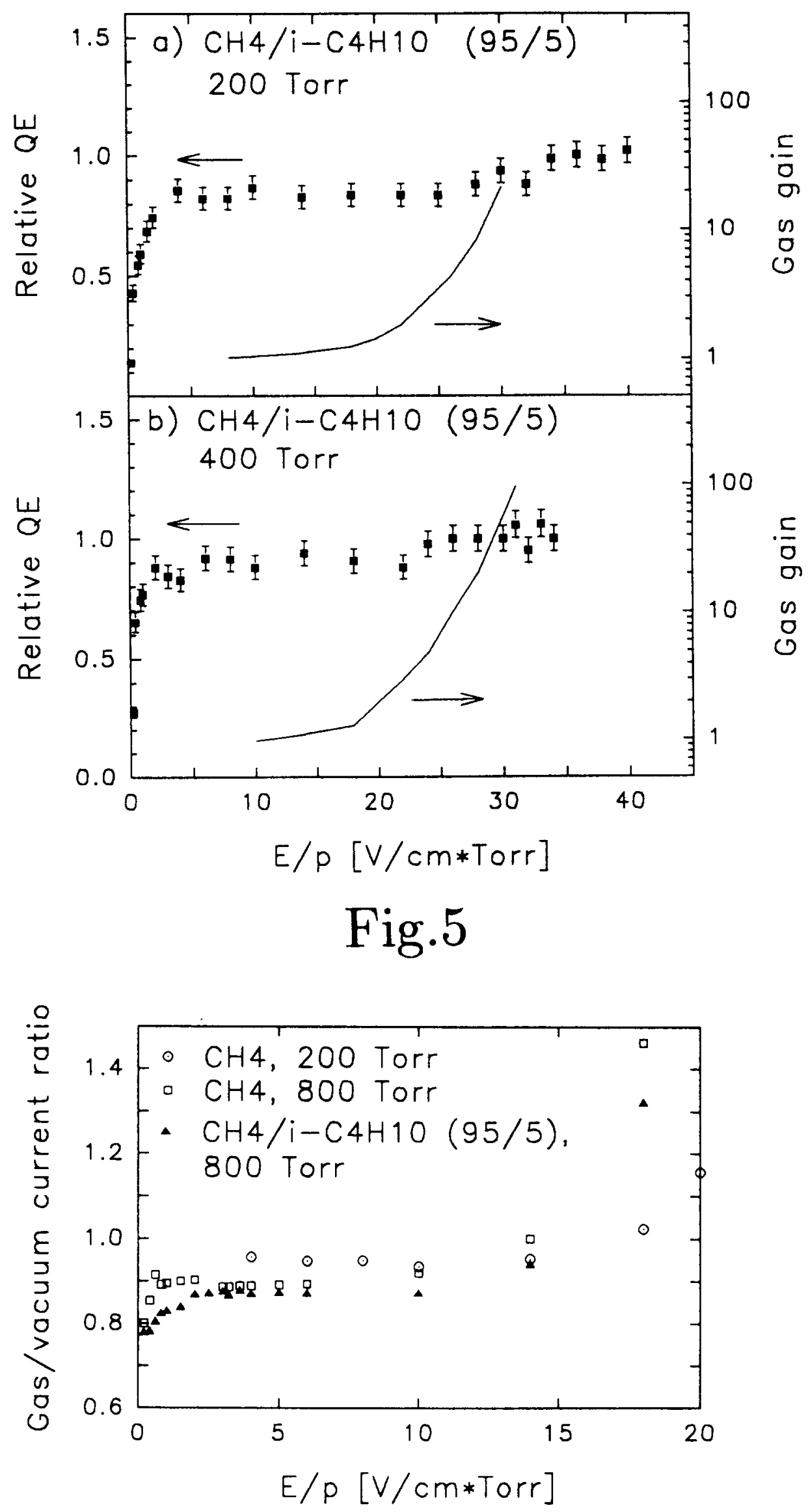

Fig.6 